\title{
A model of electron transport through a boson cavity
}

\author{
A. A. Boitsev ${ }^{1}$, J. Brasche ${ }^{2}$, H. Neidhardt ${ }^{3}$, I. Y. Popov ${ }^{1}$ \\ ${ }^{1}$ ITMO University, Kronverkskiy, 49, St. Petersburg, 197101, Russia \\ ${ }^{2}$ Institut für Mathematik, TU Clausthal, Erzstr. 1, D-38678 Clausthal-Zellerfeld, Germany \\ ${ }^{3}$ Weierstrass Institute, Mohrenstr. 39, D-10117 Berlin, Germany \\ boitsevanton@gmail.com, johannes.brasche@tu-clausthal.de, \\ hagen.neidhardt@wias-berlin.de,popov1955@gmail.com
}

PACS 85.60.Jb, 88.40.H, 85.35.Be, 73.63.Kv, 42.50.Pq

DOI 10.17586/2220-8054-2018-9-2-171-178

We propose a model describing electronic transport through a boson cavity. We use the Jaynes-Cummings model dealing with a two-level quantum dot coupled to a quantized electro-magnetic field and two semi-infinite wires. The mathematical background of our model is given by the theory of self-adjoint extensions of symmetric operators. Using the boundary triplets approach, the gamma-field and the Weyl function were calculated. In addition, we obtained the scattering matrix for the model system.

Keywords: boundary triplets, extensions, scattering, solvable model.

Received: 21 February 2018

Revised: 1 March 2018

\section{Introduction}

To study the steady state current flowing through a quantum device in the framework of Landauer-Büttiker approach, it is necessary to consider a quantum system as an inner system (quantum dot) with left and right leads attached to it, i.e. free-fermion reservoirs with two different electro-chemical potentials. The goal was to calculate the steady electron current going from one lead through the dot the other one. This current is directly related to the transmission coefficients of some natural scattering system related to this particle transport problem. This, approach was justified a few years ago [1-3]. It, effectively, reduces the problem to investigation of a one-particle Hamiltonian.

In the present paper we consider the Jaynes-Cummings model [4,5]. The Jaynes-Cummings model serves to determine how quantization of the radiation field affects the predictions for the evolution of the state of a two-level system in comparison with semi-classical theory of light-atom interaction. It is often applied to description of the interaction between an atom and a laser field [6]. We used a version suggested in [7,8]. More precisely, the authors of $[7,8]$ use the discrete Hamiltonian. In contrast, we use the continuous Hamiltonian. Namely, we have a two-level quantum dot coupled, from one side, to the standard Jaynes-Cummings one-mode photon resonator, and from the other side to two semi-infinite leads. To construct the model, we use the operator extensions theory approach (see, e.g., [9-11] and references in [12]) in the framework of boundary triplet approach (see, e.g., [13-16]). Detailed mathematical background of the model is developed in [17].

\section{Preliminaries}

\subsection{Linear relations}

A linear relation $\Theta$ in $\mathcal{H}$ is a closed linear subspace of $\mathcal{H} \oplus \mathcal{H}$. The set of all linear relations in $\mathcal{H}$ is denoted by $\widetilde{\mathcal{C}}(\mathcal{H})$. We denote also by $\mathcal{C}(\mathcal{H})$ the set of all closed linear (not necessarily densely defined) operators in $\mathcal{H}$. Identifying each operator $T \in \mathcal{C}(\mathcal{H})$ with its graph gr $(T)$, we regard $\mathcal{C}(\mathcal{H})$ as a subset of $\widetilde{\mathcal{C}}(\mathcal{H})$.

The role of the set $\widetilde{\mathcal{C}}(\mathcal{H})$ in extension theory becomes clear from Proposition 2.3. However, it's role in the operator theory is substantially motivated by the following circumstances: in contrast to $\mathcal{C}(\mathcal{H})$, the set $\widetilde{\mathcal{C}}(\mathcal{H})$ is closed with respect to taking inverse and adjoint relations $\Theta^{-1}$ and $\Theta^{*}$. The latter is given by: $\Theta^{-1}=\{\{g, f\}$ : $\{f, g\} \in \Theta\}$ and:

$$
\Theta^{*}=\left\{\left(\begin{array}{c}
k \\
k^{\prime}
\end{array}\right):\left(h^{\prime}, k\right)=\left(h, k^{\prime}\right) \text { for all }\left(\begin{array}{c}
h \\
h^{\prime}
\end{array}\right) \in \Theta\right\} .
$$

A linear relation $\Theta$ is called symmetric if $\Theta \subset \Theta^{*}$ and self-adjoint if $\Theta=\Theta^{*}$. 


\subsection{Boundary triplets and proper extensions}

Let us briefly recall some basic facts on boundary triplets. Let $S$ be a densely defined closed symmetric operator with equal deficiency indices $n_{ \pm}(S):=\operatorname{dim}\left(\mathfrak{N}_{ \pm i}\right), \mathfrak{N}_{z}:=\operatorname{ker}\left(S^{*}-z\right), z \in \mathbb{C}_{ \pm}$, acting on some separable Hilbert space $\mathfrak{H}$.

\section{Definition 2.1.}

(i) A closed extension $\widetilde{S}$ of $S$ is called proper if $\operatorname{dom}(S) \subset \operatorname{dom}(\widetilde{S}) \subset \operatorname{dom}\left(S^{*}\right)$.

(ii) Two proper extensions $\widetilde{S}^{\prime}, \widetilde{S}$ are called disjoint if $\operatorname{dom}\left(\widetilde{S}^{\prime}\right) \cap \operatorname{dom}(\widetilde{S})=\operatorname{dom}(S)$ and transversal if in addition $\operatorname{dom}\left(\widetilde{S}^{\prime}\right)+\operatorname{dom}(\widetilde{S})=\operatorname{dom}\left(S^{*}\right)$.

we denote by Ext $\mathrm{E}_{\mathrm{S}}$ the set of all proper extensions of $S$ completed by the non-proper extensions $S$ and $S^{*}$ is denoted. For instance, any self-adjoint or maximal dissipative (accumulative) extension is proper.

Definition 2.2 ( [18]). A triplet $\Pi=\left\{\mathcal{H}, \Gamma_{0}, \Gamma_{1}\right\}$, where $\mathcal{H}$ is an auxiliary Hilbert space and $\Gamma_{0}, \Gamma_{1}: \operatorname{dom}\left(S^{*}\right) \rightarrow$ $\mathcal{H}$ are linear mappings, is called a boundary triplet for $S^{*}$ if the "abstract Green's identity":

$$
\left(S^{*} f, g\right)-\left(f, S^{*} g\right)=\left(\Gamma_{1} f, \Gamma_{0} g\right)-\left(\Gamma_{0} f, \Gamma_{1} g\right), f, g \in \operatorname{dom}\left(S^{*}\right) .
$$

is satisfied and the mapping $\Gamma:=\left(\Gamma_{0}, \Gamma_{1}\right)^{\top}: \operatorname{dom}\left(S^{*}\right) \rightarrow \mathcal{H} \oplus \mathcal{H}$ is surjective, i.e. $\operatorname{ran}(\Gamma)=\mathcal{H} \oplus \mathcal{H}$.

A boundary triplet $\Pi=\left\{\mathcal{H}, \Gamma_{0}, \Gamma_{1}\right\}$ for $S^{*}$ always exists whenever $n_{+}(S)=n_{-}(S)$. Note also that $n_{ \pm}(S)=\operatorname{dim}(\mathcal{H})$ and $\operatorname{ker}\left(\Gamma_{0}\right) \cap \operatorname{ker}\left(\Gamma_{1}\right)=\operatorname{dom}(S)$.

With any boundary triplet $\Pi$ one associates two canonical self-adjoint extensions $S_{j}:=S^{*} \uparrow \operatorname{ker}\left(\Gamma_{j}\right), j \in$ $\{0,1\}$. Conversely, for any extension $S_{0}=S_{0}^{*} \in$ Ext $_{\mathrm{S}}$, there exists a (non-unique) boundary triplet $\Pi=$ $\left\{\mathcal{H}, \Gamma_{0}, \Gamma_{1}\right\}$ for $S^{*}$ such that $S_{0}:=S^{*} \uparrow \operatorname{ker}\left(\Gamma_{0}\right)$.

Using the concept of boundary triplets one can parametrize all proper extensions of $A$ in the following way.

Proposition 2.3 ( [19]). Let $\Pi=\left\{\mathcal{H}, \Gamma_{0}, \Gamma_{1}\right\}$ be a boundary triplet for $S^{*}$. Then the mapping

$$
\operatorname{Ext}_{\mathrm{S}} \ni \widetilde{\mathrm{S}} \rightarrow \Gamma \operatorname{dom}(\widetilde{\mathrm{S}})=\left\{\left(\Gamma_{0} \mathrm{f}, \Gamma_{1} \mathrm{f}\right)^{\top}: \mathrm{f} \in \operatorname{dom}(\widetilde{\mathrm{S}})\right\}=: \Theta \in \widetilde{\mathcal{C}}(\mathcal{H})
$$

establishes a bijective correspondence between the sets $\operatorname{Ext}_{\mathrm{S}}$ and $\widetilde{\mathcal{C}}(\mathcal{H})$. We write $\widetilde{S}=S_{\Theta}$ if $\widetilde{S}$ corresponds to $\Theta$ by (2). Moreover, the following holds:

(i) $S_{\Theta}^{*}=S_{\Theta^{*}}$, in particular, $S_{\Theta}^{*}=S_{\Theta}$ if and only if $\Theta^{*}=\Theta$.

(ii) $S_{\Theta}$ is symmetric (self-adjoint) if and only if $\Theta$ is symmetric (self-adjoint).

(iii) The extensions $S_{\Theta}$ and $S_{0}$ are disjoint (transversal) if and only if there is a closed (bounded) operator B such that $\Theta=\operatorname{gr}(B)$. In this case (2) takes the form

$$
S_{\Theta}:=S_{\mathrm{gr}(B)}=S^{*} \uparrow \operatorname{ker}\left(\Gamma_{1}-B \Gamma_{0}\right) .
$$

In particular, $S_{j}:=S^{*} \uparrow \operatorname{ker}\left(\Gamma_{j}\right)=S_{\Theta_{j}}, j \in\{0,1\}$, where $\Theta_{0}:=\left(\begin{array}{c}\{0\} \\ \mathcal{H}\end{array}\right)$ and $\Theta_{1}:=\left(\begin{array}{c}\mathcal{H} \\ \{0\}\end{array}\right)=\operatorname{gr}(\mathbb{O})$ where $(\mathbb{O}$ denotes the zero operator in $\mathcal{H}$. Note also that $\widetilde{\mathcal{C}}(\mathcal{H})$ contains the trivial linear relations $\{0\} \times\{0\}$ and $\mathcal{H} \times \mathcal{H}$ parameterizing the extensions $S$ and $S^{*}$, respectively, for any boundary triplet $\Pi$.

\subsection{Gamma field and Weyl function}

It is well known that Weyl function is an important tool in the direct and inverse spectral theory of SturmLiouville operators. In [19], the concept of Weyl function was generalized to the case of an arbitrary symmetric operator $S$ with $n_{+}(S)=n_{-}(S) \leq \infty$. Following [19], we briefly recall basic facts on Weyl functions and $\gamma$-fields associated with a boundary triplet $\Pi$.

Definition 2.4 ( [19]). Let $\Pi=\left\{\mathcal{H}, \Gamma_{0}, \Gamma_{1}\right\}$ be a boundary triplet for $S^{*}$ and $S_{0}=S^{*} \uparrow \operatorname{ker}\left(\Gamma_{0}\right)$. The operator valued functions $\gamma(\cdot): \rho\left(S_{0}\right) \rightarrow[\mathcal{H}, \mathcal{H}]$ and $M(\cdot): \rho\left(S_{0}\right) \rightarrow[\mathcal{H}]$ defined by:

$$
\gamma(z):=\left(\Gamma_{0} \uparrow \mathfrak{N}_{z}\right)^{-1} \quad M(z):=\Gamma_{1} \gamma(z), \quad z \in \rho\left(S_{0}\right),
$$

are called the $\gamma$-field and the Weyl function, respectively, corresponding to the boundary triplet $\Pi$.

Clearly, the Weyl function can equivalently be defined by:

$$
M(z) \Gamma_{0} f_{z}=\Gamma_{1} f_{z}, \quad f_{z} \in \mathfrak{N}_{z}, \quad z \in \rho\left(S_{0}\right) .
$$


The $\gamma$-field $\gamma(\cdot)$ and the Weyl function $M(\cdot)$ in (4) are well defined. Moreover, both $\gamma(\cdot)$ and $M(\cdot)$ are holomorphic on $\rho\left(S_{0}\right)$ and the following relations:

$$
\gamma(z)=\left(I+(z-\zeta)\left(S_{0}-z\right)^{-1}\right) \gamma(\zeta), z, \zeta \in \rho\left(S_{0}\right)
$$

and

$$
M(z)-M(\zeta)^{*}=(z-\bar{\zeta}) \gamma(\zeta)^{*} \gamma(z), z, \zeta \in \rho\left(S_{0}\right),
$$

hold. Identity (6) yields that $M(\cdot)$ is $[\mathcal{H}]$-valued Nevanlinna function $(M(\cdot) \in R[\mathcal{H}])$, i.e. $M(\cdot)$ is $[\mathcal{H}]$-valued holomorphic function on $\mathbb{C}_{ \pm}$satisfying:

$$
M(z)=M(\bar{z})^{*} \quad \text { and } \quad \frac{\operatorname{Im}(M(z))}{\operatorname{Im}(z)} \geq 0, z \in \mathbb{C}_{+} \cup \mathbb{C}_{-} .
$$

It follows also from (6) that $0 \in \rho(\operatorname{Im}(M(z)))$ for all $z \in \mathbb{C}_{ \pm}$.

\subsection{Krein-type formula for resolvents}

Let $\Pi=\left\{\mathcal{H}, \Gamma_{0}, \Gamma_{1}\right\}$ be a boundary triplet for $S^{*}, M(\cdot)$ and $\gamma(\cdot)$ the corresponding Weyl function and $\gamma$-field, respectively. For any proper (not necessarily self-adjoint) extension $\widetilde{S}_{\Theta} \in$ Ext $_{\mathrm{S}}$ with non-empty resolvent set $\rho\left(\widetilde{S}_{\Theta}\right)$ the following Krein-type formula holds (cf. [19]):

$$
\left(S_{\Theta}-z\right)^{-1}-\left(S_{0}-z\right)^{-1}=\gamma(z)(\Theta-M(z))^{-1} \gamma^{*}(\bar{z}), z \in \rho\left(S_{0}\right) \cap \rho\left(S_{\Theta}\right) .
$$

Formula (7) extends the known Krein formula for canonical resolvents to the case of any $S_{\Theta} \in$ Ext $_{S}$ with $\rho\left(S_{\Theta}\right) \neq \emptyset$. Moreover, due to relations (2), (3) and (4) formula (7) is related to the boundary triplet $\Pi$. We emphasize, that this relation makes it possible to apply the Krein-type formula (7) to boundary value problems (see, e.g., [14] and references in [12]).

\subsection{Scattering and Weyl function}

Let $S$ be a densely defined closed symmetric operator with finite equal deficiency indices $n_{ \pm}(S)$ and $\Pi=$ $\left\{\mathcal{H}, \Gamma_{0}, \Gamma_{1}\right\}$ is a boundary triplet for $S^{*}$, Let $S_{0}=S^{*}\left\lceil\operatorname{ker} \Gamma_{0}\right.$ and $S_{\Theta}$ is a self-adjoint extension corresponding to $\Theta \in \tilde{C}(\mathcal{H})$. As $\operatorname{dim} \mathcal{H}$ is finite, by (7) $\left(S_{\Theta}-z\right)^{-1}-\left(S_{0}-z\right)^{-1}$ is a finite rank operator and the system $\left\{S_{\Theta}, S_{0}\right\}$ is a so-called complete scattering system, i.e. the wave operators:

$$
W_{ \pm}\left(S_{\Theta}, S_{0}\right)=s-\lim _{t \rightarrow \pm \infty} e^{i t S_{\Theta}} e^{-i t S_{0}} P^{a c}\left(S_{0}\right)
$$

exists and they are complete, i.e. their ranges coincide with the absolutely continuous subspace $\mathfrak{H}^{a c}\left(S_{\Theta}\right)$ of $S_{\Theta}$ (see, e.g. [20], [21], [22]). By $P^{a c}\left(S_{0}\right)$ we denote the orthogonal projection on absolutely continuous subspace $\mathfrak{H}^{a c}\left(S_{0}\right)$ of $S_{0}$. The scattering operator $S\left(S_{\Theta}, S_{0}\right)$ of a scattering system $\left\{S_{\Theta}, S_{0}\right\}$ is defined as:

$$
S\left(S_{\Theta}, S_{0}\right)=W_{+}\left(S_{\Theta}, S_{0}\right)^{*} W_{-}\left(S_{\Theta}, S_{0}\right) .
$$

If we regard the scattering operator as an operator in $\mathfrak{H}^{a c}\left(S_{0}\right)$ then it becomes unitary and commutes with absolutely continuous part $S_{0}^{a c}=S_{0}\left\lceil\mathfrak{H}^{a c}\left(S_{0}\right) \cap \operatorname{dom}\left(S_{0}\right)\right.$. of $S_{0}$ and thus it is unitarily equivalent to a multiplication operator induced by a family $\left\{S_{\Theta}(z)\right\}$ of unitary operators in a spectral representation of $S_{0}^{a c}$ ( [20], Proposition 9.57). This family is called a scattering matrix of a scattering system $S\left(S_{\Theta}, S_{0}\right)$.

Since the dimension $\operatorname{dim} \mathcal{H}$ is finite, then the Weyl function $M(\cdot)$ corresponding to boundary triplet $\Pi=$ $\left\{\mathfrak{H}, \Gamma_{0}, \Gamma_{1}\right\}$ is a matrix-valued Nevanlinna function. By Fatous theorem ( [23]), the limit $M(\lambda+i 0)=\lim _{\varepsilon \rightarrow 0+0} M(\lambda+$ $i \varepsilon)$ exists for almost all $\lambda \in \mathbb{R}$. We denote the set of real point where the limit exists by $\Sigma^{M}$. We will use the notation $\mathcal{H}_{M(\lambda)}=\operatorname{ran}(M(\lambda)), \quad \lambda \in \Sigma^{M}$. By $P_{M(\lambda)}$ we will denote the orthogonal projection on $\mathcal{H}_{M(\lambda)}$. We will also use the notation $N_{\Theta}(z)=(\Theta-M(z))^{-1}, \quad z \in \mathbb{C} \backslash \mathbb{R}$, where $\Theta \in \tilde{C}(\mathcal{H})$ is a self-adjoint relation corresponding to $S_{\Theta}$. This function is well defined and the limit $N_{\Theta}(\lambda+i 0)=(\Theta-M(\lambda+i 0))^{-1}$ exists almost for every $\lambda \in \mathbb{R}$. This set we will denote as $\Sigma^{N}$.

Theorem 2.5. ( [13]) Let $S$ be a densely defined symmetric operator with finite deficiency indices in separable Hilbert space $\mathfrak{H}$, let $\Pi$ be a boundary triplet corresponding to $S^{*}$ with corresponding Weyl function $M(\cdot)$, $S_{\Theta}$ is a self-adjoint extension of $S, S_{0}=S^{*} \uparrow \operatorname{ker} \Gamma_{0}, \Theta \in \tilde{C}(\mathcal{H})$, then in $L^{2}\left(\mathbb{R}, d \lambda, \mathcal{H}_{M(\lambda)}\right)$ the scattering matrix of the complete scattering system $\left\{S_{\Theta}, S_{0}\right\}$ is given by:

$$
\mathfrak{S}_{\Theta}(\lambda)=I_{\mathcal{H}_{M(\lambda)}}+2 i \sqrt{\Im(M(\lambda+i 0))} N_{\theta}(\lambda+i 0) \sqrt{\Im(M(\lambda+i 0))}
$$

for $\lambda \in \Sigma^{M} \cap \Sigma^{N}$ 


\section{Scattering}

\subsection{Model description}

A rigorous construction of a proposed model can be found in [17]. We will show here only the main results. We consider the Hilbert space $\mathfrak{H}=L^{2}\left(\mathbb{R}_{-}\right) \oplus L^{2}\left(\mathbb{R}_{+}\right)$where $\mathbb{R}_{-}=(-\infty, 0)$ and $\mathbb{R}_{+}=(0, \infty)$. On the subspaces $\mathfrak{H}_{l}:=L^{2}\left(\mathbb{R}_{-}\right)$and $\mathfrak{H}_{r}:=L^{2}\left(\mathbb{R}_{+}\right)$we consider the closed symmetric operators $H_{l}=-\frac{d^{2}}{d x^{2}}+v_{l}$ and $H_{r}=-\frac{d^{2}}{d x^{2}}+v_{r}$. We set $\mathfrak{H}:=\mathfrak{H}_{l} \oplus \mathfrak{H}_{r}=L^{2}(\mathbb{R})$ and $H:=H_{l} \oplus H_{r}$. Operator $H$ can be regarded as the symmetric operator:

$$
A=-\frac{d^{2}}{d x^{2}}+v(x), \quad v(x):= \begin{cases}v_{l} & x \in \mathbb{R}_{-} \\ v_{r} & x \in \mathbb{R}_{+}\end{cases}
$$

with domain $\operatorname{dom}(A)=W_{0}^{2,2}(\mathbb{R}):=\left\{f \in W^{2,2}(\mathbb{R}): f(0)=f^{\prime}(0)=0\right\}$. The operator $A$ is symmetric and has deficiency indices $n_{ \pm}(A)=2$. For simplicity we assume that $0 \leq v_{r} \leq v_{l}$. It can be checked (see [17]) that the triplet $\Pi_{A}=\left\{\mathcal{H}^{A}, \Gamma_{0}^{A}, \Gamma_{1}^{A}\right\}$ with:

$$
\mathcal{H}^{A}:=\begin{array}{cc}
\mathcal{H}^{H_{l}} & \mathbb{C} \\
\underset{\mathcal{H}^{H_{r}}}{\oplus}= & \mathbb{C},
\end{array} \quad \Gamma_{0}^{A} f:=\left(\begin{array}{l}
f(-0) \\
f(+0)
\end{array}\right), \quad \Gamma_{1}^{A}:=\left(\begin{array}{c}
-f^{\prime}(-0) \\
f^{\prime}(+0)
\end{array}\right),
$$

defines a boundary triplet for $A^{*}$. The Weyl function $M^{A}(z)$ of the boundary triplet $\Pi_{A}$ is given by:

$$
M^{A}(z)=\left(\begin{array}{cc}
m^{H_{l}}(z) & 0 \\
0 & m^{H_{r}}(z)
\end{array}\right)=\left(\begin{array}{cc}
i \sqrt{z-v_{l}} & 0 \\
0 & i \sqrt{z-v_{r}}
\end{array}\right), \quad z \in \rho\left(A_{0}\right),
$$

where $A_{0}:=A^{*} \uparrow \operatorname{ker}\left(\Gamma_{0}^{A}\right)$. Notice that $A_{0}$ has Dirichlet boundary conditions.

We will view the point zero as a quantum dot or quantum cavity. In particular, the Hilbert space $\mathcal{H}^{A}=\mathbb{C}^{2}$ is considered to be the state space of the quantum dot and the self-adjoint operator $B$ as the Hamiltonian of the dot, where $A_{B}:=A^{*} \uparrow \operatorname{ker}\left(\Gamma_{1}^{A}-B \Gamma_{0}^{A}\right)$ is a self-adjoint extension of $A$. The Hamiltonian $B$ describes a two level system to which we are going to couple bosons. The state space of the bosons is the Hilbert space $\mathfrak{T}=l_{2}\left(\mathbb{N}_{0}\right)$, $\mathbb{N}_{0}:=\mathbb{N} \cup\{0\}$. The boson operator $T$ is given by:

$$
\begin{aligned}
T \vec{\xi} & =T\left\{\xi_{k}\right\}_{k \in \mathbb{N}_{0}}=\left\{k \xi_{k}\right\}_{k \in \mathbb{N}_{0}}, \\
\vec{\xi} & =\left\{\xi_{k}\right\}_{k \in \mathbb{N}_{0}} \in \operatorname{dom}(T):=\left\{\left\{\xi_{k}\right\}_{k \in \mathbb{N}_{0}} \in l_{2}\left(\mathbb{N}_{0}\right):\left\{k \xi_{k}\right\}_{k \in \mathbb{N}_{0}} \in l_{2}\left(\mathbb{N}_{0}\right)\right\} .
\end{aligned}
$$

The Hamiltonian $T$ describes a system of bosons which do not interact mutually. The number of bosons is not fixed and varies from zero to infinity. The Hilbert space $\mathfrak{T}$ has a natural basis given by $e_{k}=\left\{\delta_{k j}\right\}_{j \in \mathbb{N}_{0}}$. Let us introduce the creation and annihilation operator $b^{*}$ and $b$, respectively, defined by:

$$
b^{*} e_{k}=\sqrt{k+1} e_{k+1}, \quad k \in \mathbb{N}_{0}, \quad \text { and } \quad b e_{k}=\sqrt{k} e_{k-1}, \quad k \in \mathbb{N}_{0},
$$

where $e_{-1}=0$. One easily checks that $T=b^{*} b$.

In order to couple these two systems let us consider the closed symmetric operator:

$$
S:=A \otimes I_{\mathfrak{T}}+I_{\mathfrak{H}} \otimes T
$$

in the Hilbert space $\mathfrak{K}:=\mathfrak{H} \otimes \mathfrak{T}$. Setting the following:

$$
\begin{array}{ll}
\mathfrak{K}_{l}:=\mathfrak{H}_{l} \otimes \mathfrak{T}, & S_{l}:=H_{l} \otimes I_{\mathfrak{T}}+I_{\mathfrak{H}_{l}} \otimes T, \\
\mathfrak{K}_{r}:=\mathfrak{H}_{r} \otimes \mathfrak{T}, & S_{r}:=H_{r} \otimes I_{\mathfrak{T}}+I_{\mathfrak{H}_{r}} \otimes T,
\end{array}
$$

we obtain:

$$
\mathfrak{K}=\mathfrak{K}_{l} \oplus \mathfrak{K}_{r} \quad \text { and } \quad S=S_{l} \oplus S_{r} .
$$

The corresponding Weyl function $M^{S}(\cdot)$ is given by:

$$
M^{S}(z)=\left(\begin{array}{cc}
M^{S_{l}}(z) & 0 \\
0 & M^{S_{r}}(z)
\end{array}\right), \quad z \in \rho\left(S_{0}\right), \quad S_{0}=S_{l}^{D} \oplus S_{r}^{D},
$$

where $S_{l}^{D}=H_{l}^{D} \otimes I_{\mathfrak{T}}+I_{\mathfrak{H}_{l}} \otimes T$ and $S_{r}^{D}=H_{r}^{D} \otimes I_{\mathfrak{T}}+I_{\mathfrak{H}_{l}} \otimes T-$ the extensions with the Dirichlet boundary conditions and:

$$
M^{S_{l}}(z)=\frac{m^{H_{l}}(z-T)-\operatorname{Re}\left(m^{H_{l}}(i-T)\right)}{\operatorname{Im}\left(m^{H_{l}}(i-T)\right)}, \quad z \in \mathbb{C}_{ \pm},
$$




$$
M^{S_{r}}(z)=\frac{m^{H_{r}}(z-T)-\operatorname{Re}\left(m^{H_{r}}(i-T)\right)}{\operatorname{Im}\left(m^{H_{r}}(i-T)\right)}, \quad z \in \mathbb{C}_{ \pm} .
$$

Moreover, we have:

$$
\left(\begin{array}{cc}
\sqrt{\operatorname{Im}\left(m^{H_{l}}(i-T)\right)} & 0 \\
0 & \sqrt{\operatorname{Im}\left(m^{H_{r}}(i-T)\right)}
\end{array}\right)=\frac{1}{\sqrt[4]{2}}\left(\begin{array}{cc}
Z_{l}^{-1 / 2} & 0 \\
0 & Z_{r}^{-1 / 2}
\end{array}\right)
$$

and:

$$
\left(\begin{array}{cc}
\operatorname{Re}\left(m^{H_{l}}(i-T)\right) & 0 \\
0 & \operatorname{Re}\left(m^{H_{r}}(i-T)\right)
\end{array}\right)=\left(\begin{array}{cc}
Z_{l} & 0 \\
0 & Z_{r}
\end{array}\right)
$$

where:

$$
\begin{aligned}
& Z_{l}:=\sqrt{\sqrt{I_{\mathfrak{T}}+\left(T+v_{l}\right)^{2}}+T+v_{l}}, \\
& Z_{r}:=\sqrt{\sqrt{I_{\mathfrak{T}}+\left(T+v_{r}\right)^{2}}+T+v_{r}} .
\end{aligned}
$$

\subsection{Scattering matrix}

We want to show that the limit $\lim _{\varepsilon \rightarrow 0} M^{S}(\lambda+i \varepsilon)$ exists. The candidate for this limit is the function $M^{S}(\lambda)$, $\lambda \in \mathbb{R}$.

Lemma 3.1. The operator-function $M^{S}(\lambda), \lambda \in \mathbb{R}$ is a bounded operator.

Consider:

$$
M^{S_{l}}(\lambda)=\frac{m^{H_{l}}(\lambda-T)-\operatorname{Re}\left(m^{H_{l}}(i-T)\right)}{\operatorname{Im}\left(m^{H_{l}}(i-T)\right)}=\bigoplus_{n=0}^{\infty} \frac{m^{H_{l}}(\lambda-n)-\operatorname{Re}\left(m^{H_{l}}(i-n)\right)}{\operatorname{Im}\left(m^{H_{l}}(i-n)\right)} .
$$

We then rewrite as follows:

$$
\frac{m^{H_{l}}(\lambda-n)-\operatorname{Re}\left(m^{H_{l}}(i-n)\right)}{\operatorname{Im}\left(m^{H_{l}}(i-n)\right)}=\frac{i \sqrt{\lambda-n-v_{l}}+\frac{1}{\sqrt{2}} \sqrt{\sqrt{1+\left(n+v_{l}\right)^{2}}+n+v_{l}}}{\frac{1}{\sqrt{2}}\left(\sqrt{\sqrt{1+\left(n+v_{l}\right)^{2}}+n+v_{l}}\right)^{-1}}
$$

and consider this sequence when $n>\lambda-v_{l}$, then the numerator can be rewritten as:

Notice that:

$$
\begin{aligned}
& \frac{1}{\sqrt{2}} \sqrt{\sqrt{1+\left(n+v_{l}\right)^{2}}+n+v_{l}}-\sqrt{n-\lambda+v_{l}}= \\
& \frac{1}{2} \frac{\sqrt{1+\left(n+v_{l}\right)^{2}}-\left(n+v_{l}-2 \lambda\right)}{\frac{1}{\sqrt{2}} \sqrt{\sqrt{1+\left(n+v_{l}\right)^{2}}+n+v_{l}}+\sqrt{n-\lambda+v_{l}}}
\end{aligned}
$$

$$
\frac{\sqrt{2} \sqrt{\sqrt{1+\left(n+v_{l}\right)^{2}}+n+v_{l}}}{\frac{2}{\sqrt{2}} \sqrt{\sqrt{1+\left(n+v_{l}\right)^{2}}+n+v_{l}}+\sqrt{n-\lambda+v_{l}}}
$$

has the finite limit, so it is bounded and we have to examine:

$$
\sqrt{1+\left(n+v_{l}\right)^{2}}-\left(n+v_{l}-2 \lambda\right)=\frac{1+4 n \lambda-4 \lambda^{2}+4 v_{l} \lambda}{\sqrt{1+\left(n+v_{l}\right)^{2}}+\left(n+v_{l}-2 \lambda\right)}
$$

which also has the finite limit and so bounded. Thus, the sequence is bounded for $n>\lambda-v_{l}$, so it is bounded for $n \in \mathbb{N}_{0}$. Analogously, we deal with $M^{S_{r}}$.

Lemma 3.2. $\lim _{\varepsilon \rightarrow 0} M^{S}(\lambda+i \varepsilon)=M^{S}(\lambda), \lambda \in \mathbb{R}$.

Consider:

$$
\left\|M^{S_{l}}(\lambda)-M^{S_{l}}(\lambda+i \varepsilon)\right\|=\left\|\bigoplus_{n=0}^{\infty} \frac{m^{H_{l}}(\lambda-n)-\left(m^{H_{l}}(\lambda+i \varepsilon-n)\right)}{\operatorname{Im}\left(m^{H_{l}}(i-n)\right)}\right\| .
$$


We obtain:

$$
\begin{gathered}
\left\|\frac{m^{H_{l}}(\lambda-n)-\left(m^{H_{l}}(\lambda+i \varepsilon-n)\right)}{\operatorname{Im}\left(m^{H_{l}}(i-n)\right)}\right\|=\left\|\frac{i \sqrt{\lambda-n-v_{l}}-i \sqrt{\lambda+i \varepsilon-n-v_{l}}}{\frac{1}{\sqrt{2}}\left(\sqrt{\sqrt{1+\left(n+v_{l}\right)^{2}}+n+v_{l}}\right)^{-1}}\right\|= \\
\left\|\frac{\sqrt{2} \sqrt{\sqrt{1+\left(n+v_{l}\right)^{2}}+n+v_{l}}}{\sqrt{\lambda-n-v_{l}}+\sqrt{\lambda+i \varepsilon-n-v_{l}}}\right\| \varepsilon \leq\left\|\frac{\sqrt{2} \sqrt{\sqrt{1+\left(n+v_{l}\right)^{2}}+n+v_{l}}}{2 \sqrt{\lambda-n-v_{l}}}\right\|_{\varepsilon}
\end{gathered}
$$

The norm in the right hand side is bounded for integer $n$, so:

$$
\frac{m^{H_{l}}(\lambda-n)-\left(m^{H_{l}}(\lambda+i \varepsilon-n)\right)}{\operatorname{Im}\left(m^{H_{l}}(i-n)\right)} \rightarrow 0, \quad \varepsilon \rightarrow+0,
$$

uniformly with respect to $n$. Analogously we deal with $M^{S_{r}}$.

Let us show that the operator:

$$
\left(\Theta-M^{S}(\lambda+i \varepsilon)\right)^{-1}
$$

exists. It is sufficient to show that $\left(\operatorname{Im}\left(M^{S}(\lambda)\right)\right)^{-1}$ exists.

Lemma 3.3. $\left(\operatorname{Im}\left(M^{S}(\lambda)\right)\right)^{-1}$ exists for $\lambda \neq n+v_{l}$ and $\lambda \neq n+v_{r}$.

Consider:

$$
\operatorname{Im}\left(M^{S_{l}}(\lambda)\right)=\frac{\operatorname{Im}\left(m^{H_{l}}(\lambda-T)\right)}{\operatorname{Im}\left(m^{H_{l}}(i-T)\right)}=\bigoplus_{n=0}^{\infty} \frac{\operatorname{Im}\left(i \sqrt{\lambda-n-v_{l}}\right)}{\operatorname{Im}\left(m^{H_{l}}(i-n)\right)}=\bigoplus_{n=0}^{\infty} \frac{\operatorname{Re}\left(\sqrt{\lambda-n-v_{l}}\right)}{\operatorname{Im}\left(m^{H_{l}}(i-n)\right)}
$$

and $\operatorname{Re}\left(\sqrt{\lambda-n-v_{l}}\right)=0$ when $n \geq \lambda-v_{l}$ and $\operatorname{Re}\left(\sqrt{\lambda-n-v_{l}}\right)=\sqrt{\lambda-n-v_{l}}$ when $n<\lambda-v_{l}$. If $\lambda \neq n+v_{l}$ then:

$$
\operatorname{Im}\left(M^{S_{l}}(\lambda)\right)^{-1}=\bigoplus_{0}^{n<\lambda-v_{l}} \frac{\operatorname{Im}\left(m^{H_{l}}(i-n)\right)}{\sqrt{\lambda-n-v_{l}}} .
$$

Analogously, we deal with $M^{S_{r}}$.

For scattering matrix description, we need to calculate $\sqrt{\operatorname{Im}(M(\lambda+0 i))}=\sqrt{\operatorname{Im}(M(\lambda))}$.

Lemma 3.4. Operator $\sqrt{\operatorname{Im}(M(\lambda))}$ has the following form:

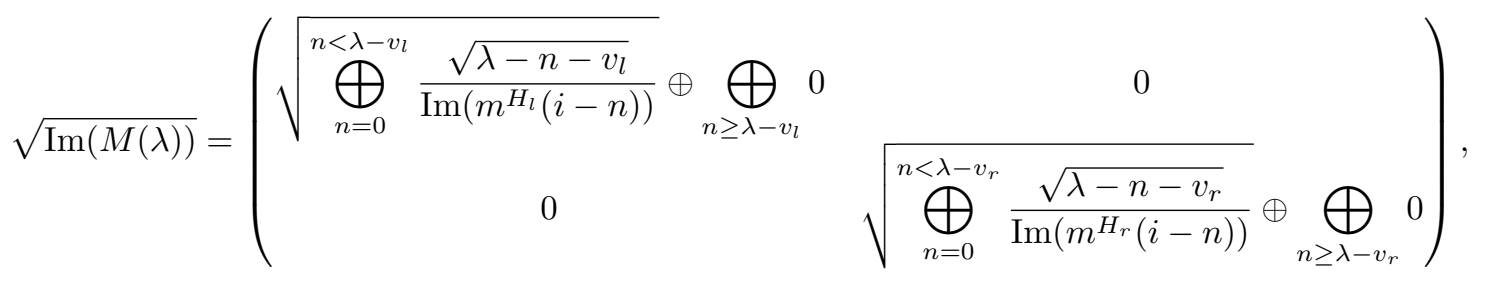

$$
\begin{aligned}
& \operatorname{Im}\left(M^{S_{l}}(\lambda)\right)=\operatorname{Im}\left(\frac{m^{H_{l}}(\lambda-T)-\operatorname{Re}\left(m^{H_{l}}(i-T)\right)}{\operatorname{Im}\left(m^{H_{l}}(i-T)\right)}\right)=\frac{\operatorname{Im}\left(m^{H_{l}}(\lambda-T)\right)}{\operatorname{Im}\left(m^{H_{l}}(i-T)\right)} .
\end{aligned}
$$

Now, as far as $m^{H_{l}}=i \sqrt{z-v_{l}}$, we obtain:

$$
\frac{\operatorname{Im}\left(m^{H_{l}}(\lambda-T)\right)}{\operatorname{Im}\left(m^{H_{l}}(i-T)\right)}=\bigoplus_{n=0}^{\infty} \frac{\operatorname{Im}\left(m^{H_{l}}(\lambda-n)\right)}{\operatorname{Im}\left(m^{H_{l}}(i-n)\right)}=\bigoplus_{n=0}^{\infty} \frac{\operatorname{Im}\left(i \sqrt{\lambda-n-v_{l}}\right)}{\operatorname{Im}\left(m^{H_{l}}(i-n)\right)} .
$$

As far as when $n<\lambda-v_{l}$ we have $\left.\operatorname{Im}\left(i \sqrt{\lambda-n-v_{l}}\right)=\sqrt{\lambda-n-v_{l}}\right)$ and when $n \geq \lambda-v_{l}$ we have $\operatorname{Im}\left(i \sqrt{\lambda-n-v_{l}}\right)=0$, we obtain that:

$$
\frac{\operatorname{Im}\left(m^{H_{l}}(\lambda-T)\right)}{\operatorname{Im}\left(m^{H_{l}}(i-T)\right)}=\bigoplus_{n=0}^{n<\lambda-v_{l}} \frac{\sqrt{\lambda-n-v_{l}}}{\operatorname{Im}\left(m^{H_{l}}(i-n)\right)} .
$$


Then,

$$
\operatorname{Im}\left(M^{S}(\lambda)\right)=\left(\begin{array}{ccc}
\bigoplus_{n=0}^{n<\lambda-v_{l}} \frac{\sqrt{\lambda-n-v_{l}}}{\operatorname{Im}\left(m^{H_{l}}(i-n)\right)} & \bigoplus_{n \geq \lambda-v_{l}} 0 & 0 \\
0 & & \bigoplus_{n=0}^{n<\lambda-v_{r}} \frac{\sqrt{\lambda-n-v_{r}}}{\operatorname{Im}\left(m^{H_{r}}(i-n)\right)} \oplus \underset{n \geq \lambda-v_{r}}{\bigoplus} 0
\end{array}\right)
$$

and

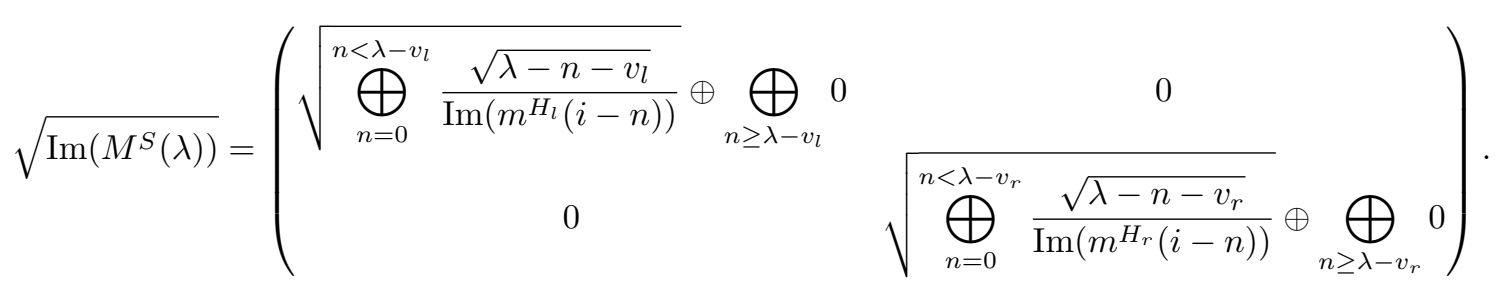

Let $\Theta$ be a matrix of the form:

$$
\Theta=\left(\begin{array}{ccc}
\bigoplus_{n=0}^{\infty}\left(\begin{array}{cc}
\alpha_{n} & \gamma_{n} \\
\bar{\gamma}_{n} & \beta_{n}
\end{array}\right) & 0 & \\
0 & \bigoplus_{n=0}^{\infty}\left(\begin{array}{ll}
\kappa_{n} & \eta_{n} \\
\bar{\eta}_{n} & \omega_{n}
\end{array}\right)
\end{array}\right)
$$

Then $\Theta-M(\lambda)$ is a block-diagonal matrix with the first block of the form

$$
\bigoplus_{n=0}^{\infty}\left(\begin{array}{cc}
\alpha_{n}-\frac{i \sqrt{\lambda-2 n-v_{l}}-\operatorname{Re}\left(m^{H_{l}}(i-2 n)\right)}{\operatorname{Im}\left(m^{H_{l}}(i-2 n)\right)} & \gamma_{n} \\
\bar{\gamma}_{n} & \beta_{n}-\frac{i \sqrt{\lambda-2 n-1-v_{l}}-\operatorname{Re}\left(m^{H_{l}}(i-2 n-1)\right)}{\operatorname{Im}\left(m^{H_{l}}(i-2 n-1)\right)}
\end{array}\right)
$$

and the second block of the form:

$$
\bigoplus_{n=0}^{\infty}\left(\begin{array}{cc}
\kappa_{n}-\frac{i \sqrt{\lambda-2 n-v_{l}}-\operatorname{Re}\left(m^{H_{r}}(i-2 n)\right)}{\operatorname{Im}\left(m^{H_{r}}(i-2 n)\right)} & \eta_{n} \\
\bar{\eta}_{n} & \omega_{n}-\frac{i \sqrt{\lambda-2 n-1-v_{l}}-\operatorname{Re}\left(m^{H_{r}}(i-2 n-1)\right)}{\operatorname{Im}\left(m^{H_{r}}(i-2 n-1)\right)}
\end{array}\right) .
$$

Let:

and:

$$
\begin{gathered}
\Delta_{1}(n)=\left(\alpha_{n}-\frac{i \sqrt{\lambda-2 n-v_{l}}-\operatorname{Re}\left(m^{H_{l}}(i-2 n)\right)}{\operatorname{Im}\left(m^{H_{l}}(i-2 n)\right)}\right) \times \\
\left(\beta_{n}-\frac{i \sqrt{\lambda-2 n-1-v_{l}}-\operatorname{Re}\left(m^{H_{l}}(i-2 n-1)\right)}{\operatorname{Im}\left(m^{H_{l}}(i-2 n-1)\right)}\right)-\gamma_{n} \bar{\gamma}_{n}
\end{gathered}
$$

$$
\begin{gathered}
\Delta_{2}(n)=\left(\kappa_{n}-\frac{i \sqrt{\lambda-2 n-v_{l}}-\operatorname{Re}\left(m^{H_{r}}(i-2 n)\right)}{\operatorname{Im}\left(m^{H_{r}}(i-2 n)\right)}\right) \times \\
\left(\omega_{n}-\frac{i \sqrt{\lambda-2 n-1-v_{l}}-\operatorname{Re}\left(m^{H_{r}}(i-2 n-1)\right)}{\operatorname{Im}\left(m^{H_{r}}(i-2 n-1)\right)}\right)-\eta_{n} \bar{\eta}_{n} .
\end{gathered}
$$

Theorem 3.5. The operator $\left(\Theta-M^{S}(\lambda+i \varepsilon)\right)^{-1}$ exists. It has the block structure with the first block of the form:

$$
\bigoplus_{n=0}^{\infty} \frac{1}{\Delta_{1}(n)}\left(\begin{array}{cc}
\beta_{n}-\frac{i \sqrt{\lambda-2 n-1-v_{l}}-\operatorname{Re}\left(m^{H_{l}}(i-2 n-1)\right)}{\operatorname{Im}\left(m^{H_{l}}(i-2 n-1)\right)} & -\gamma_{n} \\
-\bar{\gamma}_{n} & \alpha_{n}-\frac{i \sqrt{\lambda-2 n-v_{l}}-\operatorname{Re}\left(m^{H_{l}}(i-2 n)\right)}{\operatorname{Im}\left(m^{H_{l}}(i-2 n)\right)}
\end{array}\right)
$$

and the second block of the form:

$$
\bigoplus_{n=0}^{\infty} \frac{1}{\Delta_{2}(n)}\left(\begin{array}{cc}
\omega_{n}-\frac{i \sqrt{\lambda-2 n-1-v_{l}}-\operatorname{Re}\left(m^{H_{r}}(i-2 n-1)\right)}{\operatorname{Im}\left(m^{H_{r}}(i-2 n-1)\right)} & -\eta_{n} \\
-\bar{\eta}_{n} & \kappa_{n}-\frac{i \sqrt{\lambda-2 n-v_{l}}-\operatorname{Re}\left(m^{H_{r}}(i-2 n)\right)}{\operatorname{Im}\left(m^{H_{r}}(i-2 n)\right)}
\end{array}\right) .
$$


The expression for the scattering matrix is given in Theorem 2.5 .

\section{Conclusion}

An extended Jaynes-Cummings model using the ideas of [8] is constructed. It is based on the theory of self-adjoint extensions of symmetric operators. The theory gives one a rigorous mathematical methodology for introducing coupling between bosons (photons), two-level quantum dot (quantum resonator) and two semi-infinite wires. Boundary triplets, gamma-field and Krein Q-functions are found. We obtain the Weyl function. This allows us to determine the scattering matrix. Mathematical details of the model are thoroughly investigated in [17].

\section{Acknowledgements}

This work was partially financially supported by grant 16-11-10330 of Russian Science Foundation, by DFG Grants BR 1686/3-1, NE 1439/3-1. A. A. B. and I. Y.P. thank Professor J. Brasche and Clausthal University of Technology for kind hospitality.

\section{References}

[1] Aschbacher W., Jaksic V., Pautrat Y., Pillet C.-A. Transport properties of quasi-free fermions. J. Math. Phys., 2007, 48(3), P. 032101.

[2] Cornean H.D., Neidhardt H., Wilhelm L., Zagrebnov V.A. The Cayley transform applied to non-interacting quantum transport. J. Funct. Anal., 2014, 266(3), P. 14211475.

[3] Nenciu G. Independent electron model for open quantum systems: Landauer-Bttiker formula and strict positivity of the entropy production. J. Math. Phys., 48(3), P. 033302.

[4] Gerry C., Knight P. Introductory Quantum Optics. 2005. Cambridge University Press, Cambridge.

[5] Cummings F.W. Reminiscing about thesis work with E.T. Jaynes at Stanford in the 1950s. Journal of Physics B: Atomic, Molecular and Optical Physics, 2013, 46(22), P. 220202.

[6] Rodriguez-Lara B., Moya-Cessa H., Klimov A. Combining Jaynes-Cummings and anti-Jaynes-Cummings dynamics in a trapped-ion system driven by a laser. Physical Review A, 2005, 71(2), P. 023811.

[7] Neidhardt H., Wilhelm L., Zagrebnov V.A. A new model of quantum dot light emitting-absorbing devices. J. Math. Phys. Anal. Geom., 2014, 10(3), P. 350-385.

[8] Neidhardt H., Wilhelm L., Zagrebnov V.A. A new model for quantum dot light emitting-absorbing devices: proofs and supplements. Nanosystems: Physics, Chemistry, Mathematics, 2015, 6(1), P. 6-45.

[9] Albeverio S., Gesztesy F., Hoegh-Krohn R., Holden H. with an appendix by P. Exner, Solvable Models in Quantum Mechanics, second ed., AMS Chelsea Publishing, Providence, 2005.

[10] Pavlov B.S., The theory of extensions and explicity-solvable models, Russ. Math. Surv., 1987, 42(6), P. 127-168.

[11] Albeverio S., Kurasov P. Singular perturbations of differential operators. Solvable Schrodinger type operators (London Mathematical Society Lecture Notes 271), Cambridge Univ. Press, Cambridge. 2000.

[12] Popov I.Y., Kurasov P.A., Naboko S.N., Kiselev A.A., Ryzhkov A.E., Yafyasov A.M., Miroshnichenko G.P., Karpeshina Yu.E., Kruglov V.I., Pankratova T.F., Popov A.I.: A distinguished mathematical physicist Boris S. Pavlov. Nanosystems: Phys. Chem. Math. 2016, 7, P. $782-788$.

[13] Behrndt J., Malamud M.M., Neidhardt H. Scattering matrices and Weyl function. Proc. London Math. Soc., 2008, 97(3), P. $568-598$.

[14] Boitsev A.A., Neidhardt H., Popov I.Y. Dirac operator coupled to bosons. Nanosystems: Phys. Chem. Math., 2016, 7, P. $332-339$.

[15] Behrndt J., Langer M., Lotoreichik V. Boundary triples for Schrödinger operators with singular interactions on hypersurfaces. Nanosystems: Phys. Chem. Math., 2016, 7, P. 290-302.

[16] Mantile A., Posilicano A. Laplacians with singular perturbations supported on hypersurfaces. Nanosystems: Phys. Chem. Math., 2016, 7, P. $315-323$.

[17] Boitsev A.A., Brasche J.F., Malamud M.M., Neidhardt H., Popov I.Yu. Boundary triplets, tensor products and point contacts to reservoirs. arXiv:1710.07525v1 [math-ph], 2017, 62 pages (accepted in Ann. Henri Poincare).

[18] Derkach V.A., Malamud M.M. Generalized resolvents and the boundary value problems for Hermitian operators with gaps. J. Funct. Anal., 1991, 95(1), P. 1-95.

[19] Malamud M.M. Some classes of extensions of a Hermitian operator with lacunae, Ukraïn. Mat. Zh., 1992, 44(2), P. $215-233$.

[20] Baumgartel H., Wollenberg M. Mathematical Scattering Theory. Akademie-Verlag, Berlin, 1983.

[21] Kato T. Perturbation Theory for Linear Operators. Springer-Verlag New York, Inc., New York, 1966.

[22] Yafaev D.R. Mathematical Scattering Theory: General Theory. Translations of Mathematical Monographs, 105. American Mathematical Society, Providence, RI, 1992.

[23] Donoghue W.F. Monotone Matrix Functions and Analytic Continuation. Springer Verlag, Berlin and New York, 1974. 